\title{
Lipoprotein(a) concentration is associated with plasma arachidonic acid in subjects with familial hypercholesterolaemia
}

\author{
Ingunn Narverud ${ }^{1,2 *}$, Martin P. Bogsrud ${ }^{2,3}$, Linn K. L. Øyri ${ }^{1}$, Stine M. Ulven ${ }^{1}$, Kjetil Retterstøl ${ }^{1,4}$, \\ Thor Ueland $^{5,6,7}$, Monique Mulder ${ }^{8}$, Jeanine Roeters van Lennep ${ }^{8}$, Bente Halvorsen ${ }^{5,6,7}$, Pål Aukrust ${ }^{5,6,7,9}$, \\ Marit B. Veierød ${ }^{10}$ and Kirsten B. Holven ${ }^{1,2}$ \\ ${ }^{1}$ Department of Nutrition, Institute of Basic Medical Sciences, University of Oslo, Oslo, Norway \\ ${ }^{2}$ Norwegian National Advisory Unit on Familial Hypercholesterolemia, Department of Endocrinology, Morbid Obesity and \\ Preventive Medicine, Oslo University Hospital, Oslo, Norway \\ ${ }^{3}$ Unit for Cardiac and Cardiovascular Genetics, Oslo University Hospital, Oslo, Norway \\ ${ }^{4}$ Lipid Clinic, Oslo University Hospital, Oslo, Norway \\ ${ }^{5}$ Research Institute for Internal Medicine, Oslo University Hospital, Oslo, Norway \\ ${ }^{6}$ Institute of Clinical Medicine, University of Oslo, Oslo, Norway \\ ${ }^{7}$ K.G. Jebsen Inflammatory Research Center, Oslo, Norway \\ ${ }^{8}$ Department of Internal Medicine, Erasmus University Medical Center, Rotterdam, The Netherlands \\ ${ }^{9}$ Section of Clinical Immunology and Infectious Diseases, Oslo University Hospital, Oslo, Norway \\ ${ }^{10}$ Department of Biostatistics, Oslo Center for Biostatistics and Epidemiology, Institute of Basic Medical Sciences, University of \\ Oslo, Oslo, Norway
}

(Submitted 21 December 2018 - Final revision received 29 May 2019-Accepted 27 June 2019; First published online 2 July 2019)

\section{Abstract}

Elevated lipoprotein(a) (Lp(a)) is associated with CVD and is mainly genetically determined. Studies suggest a role of dietary fatty acids (FA) in the regulation of $\mathrm{Lp}(\mathrm{a})$; however, no studies have investigated the association between plasma Lp(a) concentration and $n$ - 6 FA. We aimed to investigate whether plasma Lp(a) concentration was associated with dietary $n-6$ FA intake and plasma levels of arachidonic acid (AA) in subjects with familial hypercholesterolaemia (FH). We included FH subjects with ( $n$ 68) and without ( $n$ 77) elevated Lp(a) defined as $\geq 75 \mathrm{nmol} / 1$ and healthy subjects ( $n$ 14). Total FA profile was analysed by GC-flame ionisation detector analysis, and the daily intake of macronutrients (including the sum of $n-6$ FA: $18: 2 n-6,20: 2 n-6,20: 3 n-6$ and $20: 4 n-6)$ were computed from completed FFQ. FH subjects with elevated Lp(a) had higher plasma levels of AA compared with FH subjects without elevated Lp(a) $(P=0.03)$. Furthermore, both FH subjects with and without elevated Lp(a) had higher plasma levels of AA compared with controls $(P<0 \cdot 001)$. The multivariable analyses showed associations between dietary $n$ - 6 FA intake and plasma levels of AA $(P=0.02)$ and between plasma levels of $\mathrm{Lp}(\mathrm{a})$ and AA $(P=0.006)$. Our data suggest a novel link between plasma Lp(a) concentration, dietary $n-6$ FA and plasma AA concentration, which may explain the small diet-induced increase in Lp(a) levels associated with lifestyle changes. Although the increase may not be clinically relevant, this association may be mechanistically interesting in understanding more of the role and regulation of $\mathrm{Lp}(\mathrm{a})$.

\section{Keywords: Lipoprotein(a): Plasma fatty acids: Dietary n-6 PUFA: Familial hypercholesterolaemia}

Lipoprotein(a) (Lp(a)) is an LDL-like particle bound to apolipoprotein $(a)^{(1)}$. Increasing evidence supports elevated $L p(a)$, which may be defined as $>75 \mathrm{nmol} / 1$ or $30 \mathrm{mg} / \mathrm{dl}$, for being an independent and important risk factor for $\mathrm{CVD}^{(2)}$. The plasma level of $\mathrm{Lp}(\mathrm{a})$ is considered to be determined mostly by the $L P A$ gene locus ${ }^{(3)}$; however, lifestyle-induced changes in the plasma level of $\mathrm{Lp}(\mathrm{a})$ have been reported ${ }^{(4-8)}$.
Dietary fatty acids (FA) differently impact CVD risk through divergent effects on the lipid profile ${ }^{(9)}$. Despite a large body of evidence documenting the beneficial effect of replacing SFA with PUFA in the prevention of $\mathrm{CVD}^{(10,11)}$, recent data from Chowdhury et al. and Ramsden et al. have reported conflicting results and concluded that there is no clear support for replacing SFA with PUFA ${ }^{(12,13)}$. However, in 2017, the American Heart

Abbreviations: AA, arachidonic acid; D6D, $\Delta$-6 desaturase; E \%, energy percentage; FA, fatty acid; FH, familial hypercholesterolaemia; Lp(a), lipoprotein(a).

* Corresponding author: I. Narverud, email Ingunn.narverud@medisin.uio.no 
Association presidential advisory on dietary fats and CVD strongly concluded that the incidence of CVD will be reduced when the intake of SFA is replaced with PUFA ${ }^{(14)}$. Nevertheless, one of the cornerstones in diet recommendations when performing lifestyle changes and in the prevention of CVD is replacing SFA with PUFA ${ }^{(15)}$. The essential $n$-6 PUFA linoleic acid is the predominant dietary PUFA, and lower tissue/blood concentrations of linoleic acid have been shown to be inversely associated with CVD risk $^{(16)}$ and mortality ${ }^{(17)}$. Furthermore, Marklund et al. recently concluded that higher circulating/tissue levels of linoleic acid and possibly arachidonic acid (AA) were inversely associated with the risk of major cardiovascular events after analysing thirty prospective studies from thirteen countries $^{(18)}$. However, some inconsistent data have also been reported. In the reanalysis of the Sydney Diet Heart study, it was shown that the intervention group had higher rates of death than controls ${ }^{(19)}$; and in a Mendelian randomisation study, genetically predicted linoleic acid was shown not to be associated with ischemic heart disease but was associated with lower diabetes risk $^{(20)}$. Recently, Berk et al. found increased Lp(a) levels elicited by diet-induced weight loss after a low-fat diet in overweight and obese subjects ${ }^{(4)}$. However, they found no change in Lp(a) levels after bariatric surgery ${ }^{(4)}$, suggesting a role of dietary FA in the regulation of $\operatorname{Lp}(\mathrm{a})$, rather than weight loss per se. Indeed, randomised controlled trials (RCT) have found $\mathrm{Lp}(\mathrm{a})$-increasing effects of low-fat $v$. low carbohydrate diets ${ }^{(5-8)}$.

Subjects with familial hypercholesterolaemia (FH) are characterised by increased plasma levels of total and LDL-cholesterol, accelerated atherosclerosis and increased risk of premature $\mathrm{CVD}^{(21,22)}$ and may have higher $\mathrm{Lp}(\mathrm{a})$ levels than unaffected relatives ${ }^{(23)}$. Thus, FH subjects may serve as a suitable human model to investigate the underlying mechanisms of $\mathrm{Lp}(\mathrm{a})$ modification.

To our knowledge, no studies have investigated whether there is an association between Lp(a) levels and n-6 PUFA. Although the magnitude of the effect may not be clinically relevant, this association may be mechanistically interesting in understanding more of the role and the regulation of $\mathrm{Lp}(\mathrm{a})$. We aimed to investigate whether plasma $\mathrm{Lp}(\mathrm{a})$ concentration was associated with dietary $n-6$ FA intake and plasma levels of AA in subjects with $\mathrm{FH}$.

\section{Methods \\ Subjects and study design}

In this cross-sectional study, we invited FH subjects ( $>18$ years of age) with or without elevated $\mathrm{Lp}(\mathrm{a})$ as defined by $\mathrm{Lp}(\mathrm{a}) \geq$ or $<75$ $\mathrm{nmol} / \mathrm{l}$, respectively, who were regularly followed up at the outpatient Lipid Clinic, Oslo University Hospital, Norway. Other inclusion criteria were a definite $\mathrm{FH}$ diagnosis as defined by a positive DNA test (genetic $\mathrm{FH}$ ) or a Dutch Lipid Clinic Network score $>8^{(21)}$ (clinical $\mathrm{FH}$ ) and willingness to give a blood sample. All FH subjects continued with their current lipid-lowering therapy during the study. Exclusion criteria were diabetes mellitus type 1 or pregnant or lactating women. The study visit was coordinated simultaneously with their next prescheduled consultation at the Lipid Clinic. Age- and sex-matched (by percentage) healthy controls were recruited among employees and friends of employees at the Department of Nutrition, University of Oslo, Norway and Oslo University Hospital, Norway. Exclusion criteria for the controls were Lp(a) levels $\geq 75 \mathrm{nmol} / \mathrm{l}$, cardiovascular or metabolic disease, use of lipidlowering therapy, severe illness such as cancer in the last 5 years or pregnant or lactating women. All participants were recruited in the period September 2016-September 2017. For all participants, a non-fasting blood sample was obtained, and weight, height and blood pressure were measured. Informed consent was obtained from all the participants. The study protocol was approved by the Regional Committee of Medical and Health Research Ethics, south-east region of Norway (no. 2015/1577) and by the Privacy Ombudsman at Oslo University Hospital. The study was conducted in accordance with the principles of the Declaration of Helsinki.

\section{Total plasma fatty acid profile, fatty acid ratios and other plasma analyses}

Plasma was obtained in EDTA tubes and kept in dark on ice until centrifugation at $4^{\circ} \mathrm{C}$ for $15 \mathrm{~min}$ before being aliquoted and stored at $-80^{\circ} \mathrm{C}$ until further analysis. We analysed total plasma FA profile by GC-flame ionisation detector analysis at the commercial laboratory Vitas Analytical Services as previously described $^{(24)}$ and showed the results as percentage of total FA. We estimated certain ratios between the product and the precursor of the individual FA in plasma ( $\gamma$-linolenic acid (18:3n-6)/ linoleic acid (18:2n-6); AA (20:4n-6)/dihomo $\gamma$-linolenic acid $(20: 3 n-6)$ and eicosadienoic acid $(20: 2 n-6) /$ linoleic acid $(18: 2 n-6))$, as described elsewhere ${ }^{(25)}$. The plasma $n-6: n-3$ ratio was calculated as the total percentage of $n$ - 6 (linoleic acid, $\gamma$-linolenic acid, eicosadienoic acid, dihomo $\gamma$-linolenic acid and AA) divided by the total percentage of $n-3$ FA ( $\alpha$-linolenic acid, EPA, $n$-3 docosapentaenoic acid and DHA). Lp(a) was analysed using an immunoturbidimetric method from Roche Diagnostics at an accredited medical laboratory, Oslo University Hospital, Rikshospitalet, Oslo, Norway (NS-EN ISO 15189 : 2007). At the same medical laboratory, other biochemical analyses were measured in plasma or serum by standard methods.

\section{Dietary intake}

Nutrient intake was recorded by a self-administered 256-item food FFQ completed according to a description, both received by mail. In case of incorrect completion, the participants were interviewed at the study visit or through phone. The FFQ is developed at the Department of Nutrition, University of Oslo, Norway, and has been validated and described in detail elsewhere $^{(26)}$. Briefly, intake frequencies (monthly, weekly or daily) and portion sizes in predefined household units were registered. Open spaces were available for description of unlisted food items, which were included in the nutrient calculations. The food database AE-14 and the software 'Kostberegningssystem' (version 7.3, 2017) were used to compute the daily intake of energy and nutrients. The food database used (AE-14) is based on the official Norwegian Food Composition Table (http://www. norwegianfoodcomp.no/), supplemented with data from calculated recipes and other databases and has a large number of FA 
Table 1. Characteristics of the participants*

(Medians and 25th-75th percentiles; frequencies and percentages)

\begin{tabular}{|c|c|c|c|c|c|c|c|c|c|c|c|c|c|}
\hline & \multicolumn{4}{|c|}{ FH subjects } & & & \multirow[b]{3}{*}{$P \dagger$} & \multirow{2}{*}{\multicolumn{3}{|c|}{ Unadjusted }} & \multirow{2}{*}{\multicolumn{3}{|c|}{ Adjusted }} \\
\hline & \multicolumn{2}{|c|}{$\begin{array}{c}\mathrm{Lp}(\mathrm{a}) \geq 75 \mathrm{nmol} / \mathrm{l} \\
\quad(n 68)\end{array}$} & \multicolumn{2}{|c|}{$\begin{array}{c}\mathrm{Lp}(\mathrm{a})<75 \mathrm{nmol} / \mathrm{l} \\
(n 77)\end{array}$} & \multicolumn{2}{|c|}{$\begin{array}{l}\text { Healthy subjects } \\
(n 14)\end{array}$} & & & & & & & \\
\hline & Median & Percentiles & Median & Percentiles & Median & Percentiles & & $P \neq$ & $P \S$ & $P \|$ & $P \neq$ & $P \S$ & $P \|$ \\
\hline \multicolumn{14}{|l|}{ Descriptives } \\
\hline Age (years) & 48 & $32-61$ & 44 & $31-59$ & 44 & $34-50$ & 0.74 & & & & & & \\
\hline Sex (female) & & & & & & & 0.92 & & & & & & \\
\hline$n$ & \multirow{2}{*}{\multicolumn{2}{|c|}{$\begin{array}{c}35 \\
51.5\end{array}$}} & \multicolumn{2}{|r|}{41} & \multicolumn{2}{|r|}{8} & & & & & & & \\
\hline$\%$ & & & \multicolumn{2}{|r|}{$53 \cdot 2$} & \multicolumn{2}{|r|}{$57 \cdot 1$} & & & & & & & \\
\hline BMI $\left(\mathrm{kg} / \mathrm{m}^{2}\right)$ & $25 \cdot 9$ & $22 \cdot 2-28 \cdot 6$ & $25 \cdot 7$ & $22 \cdot 5-29 \cdot 2$ & 21.5 & $19 \cdot 1-22 \cdot 4$ & $<0.001$ & 0.81 & $<0.001$ & $<0.001$ & 1.00 & $<0.001$ & $<0.001$ \\
\hline $\mathrm{SBP}(\mathrm{mmHg})$ & 126 & $120-1369$ & 128 & $115-137$ & 114 & $107-121$ & 0.002 & 0.94 & 0.001 & 0.001 & 1.00 & 0.003 & 0.003 \\
\hline $\mathrm{DBP}(\mathrm{mmHg})$ & 74 & $71-82^{\star *}$ & 77 & 69-82 & 72 & $66-74$ & 0.06 & & & & & & \\
\hline Current smoking & & & & & & & 0.04 & & & & & & \\
\hline$n$ & \multicolumn{2}{|r|}{7} & \multicolumn{2}{|r|}{11} & \multirow{2}{*}{\multicolumn{2}{|c|}{0}} & & & & & & & \\
\hline$\%$ & & $10 \cdot 3$ & \multicolumn{2}{|r|}{$14 \cdot 3$} & & & & & & & & & \\
\hline \multicolumn{3}{|l|}{ Genetic diagnosis } & & & & & & 1.00 & & & 1.00 & & \\
\hline$n$ & \multirow{2}{*}{\multicolumn{2}{|c|}{$\begin{array}{c}66 \\
97 \cdot 1\end{array}$}} & & 75 & & & & & & & & & \\
\hline$\%$ & & & & 97.4 & & & & & & & & & \\
\hline CVD & & & & & & & & 0.02 & & & 0.0 .02 & & \\
\hline$n$ & & 15 & & 6 & & & & & & & & & \\
\hline$\%$ & & $22 \cdot 1$ & & $7 \cdot 8$ & & & & & & & & & \\
\hline Blood biochemistry & & & & & & & & & & & & & \\
\hline Tchol (mmol/l) & 4.5 & $3.9-5.3$ & 4.7 & $4.0-5 \cdot 6$ & $5 \cdot 0$ & $4 \cdot 5-5 \cdot 3$ & 0.36 & & & & & & \\
\hline HDL-C (mmol/l) & 1.3 & $1.1-1.6$ & 1.5 & $1 \cdot 2-1 \cdot 7$ & 1.6 & $1 \cdot 3-2 \cdot 1$ & 0.08 & & & & & & \\
\hline LDL-C (mmol/l) & $2 \cdot 7$ & $2 \cdot 1-3 \cdot 3$ & $2 \cdot 8$ & $2 \cdot 3-3 \cdot 6$ & 3.0 & $2 \cdot 4-3.4$ & 0.6 & & & & & & \\
\hline $\mathrm{TAG}(\mathrm{mmol} / \mathrm{l})$ & 1.0 & $0.7-1.4$ & 1.0 & $0.7-1.6$ & 1.0 & $0.8-1.5$ & 0.9 & & & & & & \\
\hline ApoA1 (g/l) & 1.4 & $1 \cdot 3-1 \cdot 6$ & 1.4 & $1 \cdot 2-1 \cdot 6$ & 1.5 & $1 \cdot 4-1 \cdot 7$ & 0.39 & & & & & & \\
\hline ApoB $(g / l)$ & 1.0 & $0.8-1 \cdot 1$ & 0.9 & $0.8-1 \cdot 1$ & 0.8 & $0.7-1.0$ & 0.15 & & & & & & \\
\hline $\mathrm{Lp}(\mathrm{a})(\mathrm{nmol} / \mathrm{l})$ & 224 & $170-326$ & 7 & $7-20$ & 10 & $7-28$ & & & & & & & \\
\hline Glucose $(\mathrm{mmol} / \mathrm{l})$ & $5 \cdot 3$ & $5 \cdot 0-5 \cdot 8$ & $5 \cdot 2$ & $4 \cdot 9-5 \cdot 6$ & $5 \cdot 1$ & $4 \cdot 6-5 \cdot 5$ & $0 \cdot 1$ & & & & & & \\
\hline Medical treatment & & & & & & & & & & & & & \\
\hline Statins & & & & & & & & 0.94 & & & 0.94 & & \\
\hline$n$ & & 63 & & 70 & & & & & & & & & \\
\hline$\%$ & & $92 \cdot 6$ & & $90 \cdot 9$ & & & & & & & & & \\
\hline PCSK9 inhibitor & & & & & & & & 0.01 & & & 0.0 .01 & & \\
\hline$n$ & & 18 & & 8 & & & & & & & & & \\
\hline$\%$ & & 26.5 & & $10 \cdot 4$ & & & & & & & & & \\
\hline Colesevelam & & & & & & & & 0.90 & & & 0.90 & & \\
\hline$n$ & & 10 & & 13 & & & & & & & & & \\
\hline$\%$ & & 14.7 & & $16 \cdot 9$ & & & & & & & & & \\
\hline Ezetimibe & & & & & & & & 0.22 & & & 0.22 & & \\
\hline$n$ & & 49 & & 47 & & & & & & & & & \\
\hline$\%$ & & $72 \cdot 1$ & & 61 & & & & & & & & & \\
\hline Acetylsalicylic acid & & & & & & & & 0.16 & & & 0.16 & & \\
\hline$n$ & & $\begin{array}{c}25 \\
36 \cdot 8\end{array}$ & & $\begin{array}{c}19 \\
24.7\end{array}$ & & & & & & & & & \\
\hline$\%$ & & $36 \cdot 8$ & & $24 \cdot 7$ & & & & & & & & & \\
\hline
\end{tabular}

FH, familial hypercholesterolaemia; Lp(a), lipoprotein(a); SBP, systolic blood pressure; DBP, diastolic blood pressure; Tchol, total cholesterol; HDL-C, HDL-cholesterol; LDL-C, LDL-cholesterol; PCSK9, proprotein convertase subtilisin/kexin type 9.

${ }^{*} P$ values from the Mann-Whitney $U$ test are shown as unadjusted and Bonferroni adjusted correction.

$\dagger$ Kruskal-Wallis test or $\chi^{2}$ test between the three groups.

‡ Mann-Whitney test or $\chi^{2}$ test between FH subjects $\mathrm{Lp}(\mathrm{a}) \geq 75 \mathrm{nmol} / \mathrm{l}$ and $\mathrm{FH}$ subjects $\mathrm{Lp}(\mathrm{a})<75 \mathrm{nmol} / \mathrm{l}$.

$\S$ Mann-Whitney test between: $F H$ subjects $\operatorname{Lp}(\mathrm{a}) \geq 75 \mathrm{nmol} / \mathrm{l}$ and controls.

|| Mann-Whitney test between $\mathrm{FH}$ subjects $\mathrm{Lp}(\mathrm{a})<75 \mathrm{nmol} / \mathrm{l}$ and controls.

१ $n 62$.

** $n 61$.

including $n-6$ PUFA. Total dietary $n-6$ PUFA is the sum of $18: 2 n-6$, $20: 2 n-6,20: 3 n-6$ and $20: 4 n-6$.

\section{Statistics}

The Kruskal-Wallis test was used for all comparisons between the three groups as regards continuous variables. Pairwise comparisons (post hoc tests) were performed using the MannWhitney test and both unadjusted and Bonferroni adjusted corrections were presented. The results are presented as medians and 25th-75th percentiles in the tables and the text and as medians and minimum and maximum values in figures. The $\chi^{2}$ test or Fisher's exact test was used for categorical data, which are presented as frequencies and percentages in tables. Linear regression analysis was performed to study the association between the dietary intake of total $n-6$ PUFA, linoleic acid and $\mathrm{AA}$ (all in energy percentage (E \%)) and plasma AA after adjustment for potential confounding variables among $\mathrm{FH}$ patients that 
Table 2. Fatty acids $(F A)$ in plasma*

(Medians and 25th-75th percentiles)

\begin{tabular}{|c|c|c|c|c|c|c|c|c|c|c|c|c|c|}
\hline & \multicolumn{4}{|c|}{ FH subjects } & & & \multirow[b]{3}{*}{$P+$} & \multirow{2}{*}{\multicolumn{3}{|c|}{ Unadjusted }} & \multirow{2}{*}{\multicolumn{3}{|c|}{ Adjusted }} \\
\hline & \multicolumn{2}{|c|}{$\begin{array}{c}\mathrm{Lp}(\mathrm{a}) \geq 75 \mathrm{nmol} / \mathrm{l}(n \\
68)\end{array}$} & \multicolumn{2}{|c|}{$\begin{array}{c}\mathrm{Lp}(\mathrm{a})<75 \mathrm{nmol} / \mathrm{I} \\
(n 77)\end{array}$} & \multicolumn{2}{|c|}{$\begin{array}{l}\text { Healthy subjects } \\
\qquad(n 14)\end{array}$} & & & & & & & \\
\hline & Median & Percentiles & Median & Percentiles & Median & Percentiles & & $P \neq$ & $P \S$ & $P \|$ & $P \neq$ & $P \S$ & $P \|$ \\
\hline \multicolumn{14}{|l|}{$n-3$ PUFA ( $\%$ of total FA) } \\
\hline $\operatorname{ALA}(18: 3 n-3)$ & 0.62 & $0.46-0.77$ & 0.62 & $0.49-0.83$ & 0.94 & $0.73-1.12$ & $<0.001$ & 0.66 & $<0.001$ & 0.001 & 1.00 & $<0.001$ & 0.003 \\
\hline EPA $(20: 5 n-3)$ & 1.72 & $1.04-2.59$ & 1.42 & $1.07-2.08$ & 1.66 & $1.03-2.04$ & 0.60 & & & & & & \\
\hline DPA (22: $5 n-3)$ & 0.60 & $0.50-0.67$ & 0.54 & $0.49-0.61$ & 0.59 & $0.55-0.61$ & 0.10 & & & & & & \\
\hline DHA $(22: 6 n-3)$ & $2 \cdot 48$ & $1.97-3.19$ & $2 \cdot 38$ & $2 \cdot 01-2.99$ & 3.03 & $2.76-3.43$ & 0.02 & 0.59 & 0.02 & 0.006 & 1.00 & 0.06 & 0.02 \\
\hline \multicolumn{14}{|l|}{ MUFA ( $\%$ of total FA) } \\
\hline $\begin{array}{l}\text { Palmitoleic acid } \\
\quad(16: 1 n-7)\end{array}$ & 1.65 & $1.34-2.02$ & 1.55 & $1.26-1.90$ & $1 \cdot 33$ & $1 \cdot 17-1 \cdot 81$ & 0.27 & & & & & & \\
\hline Oleic acid (18: $1 n-9)$ & $22 \cdot 1$ & $19 \cdot 4-24.5$ & $21 \cdot 3$ & $19 \cdot 5-24 \cdot 3$ & $19 \cdot 6$ & $18 \cdot 7-20 \cdot 3$ & 0.02 & 0.69 & 0.01 & 0.04 & 1.00 & 0.03 & 0.12 \\
\hline $\begin{array}{l}\text { cis-Vaccenic acid } \\
\quad(18: 1 n-7)\end{array}$ & 1.69 & $1.53-1.88$ & 1.60 & $1.46-1.77$ & 1.40 & $1.35-1.53$ & 0.001 & 0.07 & $<0.001$ & 0.005 & 0.21 & $<0.001$ & 0.02 \\
\hline $\begin{array}{l}\text { 11-Eicosenoic acid } \\
(20: 1 n-9)\end{array}$ & 0.17 & $0.14-0.21$ & 0.17 & $0.11-0.22$ & 0.11 & $0.11-0.14$ & 0.06 & & & & & & \\
\hline \multicolumn{14}{|l|}{ SFA ( $\%$ of total FA) } \\
\hline Lauric acid (12:0) & 0.08 & $0.05-0.12$ & 0.08 & $0.06-0.12$ & 0.12 & $0.08-0.15$ & 0.29 & & & & & & \\
\hline Myristic acid (14:0) & 0.78 & $0.64-0.94$ & 0.80 & $0.64-1.02$ & 0.88 & $0.80-0.97$ & 0.26 & & & & & & \\
\hline Pentadecylic acid (15:0) & 0.17 & $0.14-0.19$ & 0.16 & $0.14-0.20$ & 0.21 & $0.19-0.22$ & 0.001 & 0.43 & $<0.001$ & 0.001 & 1.00 & $<0.001$ & 0.003 \\
\hline Palmitic acid $(16: 0)$ & $19 \cdot 3$ & $18 \cdot 4-20.5$ & $19 \cdot 6$ & $18 \cdot 9-20 \cdot 8$ & $19 \cdot 7$ & $19 \cdot 2-20 \cdot 3$ & 0.40 & & & & & & \\
\hline Stearic acid $(18: 0)$ & 7.06 & $6.73-7.56$ & $7 \cdot 00$ & $6.51-7.52$ & 6.79 & $6.47-7.04$ & 0.20 & & & & & & \\
\hline Arachidic acid $(20: 0)$ & 0.33 & $0.29-0.38$ & 0.32 & $0.28-0.36$ & 0.25 & $0.22-0.26$ & $<0.001$ & 0.4 & $<0.001$ & $<0.001$ & 1.00 & $<0.001$ & $<0.001$ \\
\hline Behenic acid $(22: 0)$ & 077 & $068-089$ & 076 & $066-083$ & 062 & $060-070$ & 0.02 & 0.61 & 0.02 & 0.06 & 1.00 & 0.06 & 0.18 \\
\hline Lignoceric acid (24:0) & 0.55 & $0.46-0.69$ & 0.56 & $0.47-0.67$ & 0.39 & $0.36-0.62$ & 0.07 & & & & & & \\
\hline
\end{tabular}

$\mathrm{FH}$, familial hypercholesterolaemia; Lp(a), lipoprotein(a); ALA, $\alpha$-linolenic acid; DPA, docosapentaenoic acid

${ }^{*} P$ values from the Mann-Whitney $U$ test are shown as unadjusted and Bonferroni adjusted correction.

$\dagger$ Kruskal-Wallis test between the three groups.

† Mann-Whitney test between FH subjects $L p(a) \geq 75 \mathrm{nmol} / \mathrm{l}$ and $\mathrm{FH}$ subjects $\mathrm{Lp}(\mathrm{a})<75 \mathrm{nmol} / \mathrm{l}$.

$\S$ Mann-Whitney test between FH subjects $L p(a) \geq 75 \mathrm{nmol} / \mathrm{l}$ and controls.

|| Mann-Whitney test between FH subjects $\mathrm{Lp}(\mathrm{a})<75 \mathrm{nmol} / \mathrm{l}$ and controls.

completed FFQ. Furthermore, logistic regression analysis was used to adjust for potential confounding variables when studying the association between plasma AA and plasma Lp(a) concentration. Results are presented as regression coefficients and OR, respectively, with $95 \% \mathrm{CI}$. The regression analyses were guided by directed acyclic graphs. Statistical analyses were performed by IBM SPSS Statistics 24.ink. $P$ values (two tailed) $<0.05$ were considered significant.

\section{Results}

\section{Characteristics}

In total, FH subjects with ( $n$ 68) and without ( $n$ 77) elevated $\mathrm{Lp}$ (a) levels defined as $\geq$ and $<75 \mathrm{nmol} / 1$ plasma $\mathrm{Lp}$ (a) and fourteen healthy controls were included in the study (online Supplementary Fig. S1). All the FH subjects had a genetically verified diagnosis except for four who had clinical $\mathrm{FH}^{(21)}$ (Table 1). All FH patients used lipid-lowering therapy and had generally received dietary counselling as part of their standard follow-up at the lipid clinic. Both groups of FH subjects had significantly higher BMI $(P>0 \cdot 001)$ and systolic blood pressure $(P=0.003)$ than the healthy controls. More FH subjects with elevated Lp(a) levels had experienced CVD $(P=0.02)$ and were more often treated with proprotein convertase subtilisin/kexin type 9 (PCSK9) inhibitors ( $P=0 \cdot 01)$ than the FH subjects without elevated Lp(a). Although non-fasting blood samples were taken, median TAG levels were not elevated according to the European guidelines, where TAG levels $\geq 1.7 \mathrm{mmol} / 1$ is considered elevated $^{(27)}$. However, $19 \cdot 1,19 \cdot 5$ and $14 \cdot 3 \%$ of the FH subjects with elevated Lp(a), FH subjects without elevated Lp(a) and controls had TAG levels $\geq 1.7 \mathrm{mmol} / 1$, respectively.

\section{Plasma fatty acid profile and ratios of fatty acids}

For plasma levels of $n$ - 6 PUFA, we found several significant differences between the groups (Fig. 1). First, we observed higher level of AA $(20: 4 n-6)$ in FH subjects with elevated Lp(a) compared with $\mathrm{FH}$ subjects without elevated $\mathrm{Lp}(\mathrm{a})$ $(P=0 \cdot 03$; Fig. 1(a)). Furthermore, the levels of AA $(20: 4 n-6)$, dihomo- $\gamma$-linolenic acid $(20: 3 n-6)$ and $\gamma$-linolenic acid $(18: 3 n-6)$ were all higher in both $\mathrm{FH}$ groups compared with the healthy controls $(P<0.001$ for all; Fig. 1(a), (b) and (c), respectively). Contrary, the level of linoleic acid (18:2n-6) was lower in both $\mathrm{FH}$ groups compared with the healthy subjects ( $P<0.001$ for both; Fig. 1(d)), and the level of eicosadienoic acid (20:2n-6) was lower in FH with elevated Lp(a) compared with healthy subjects $(P<0 \cdot 01$; Fig. 1(e)). For the plasma levels of $n-3$ PUFA (Table 2), we found lower levels of $\alpha$-linolenic acid $(18: 3 n-3)$ in both FH groups compared with the healthy controls $(P<0.005$ for both). The level of DHA (22:6n-3) was lower in 
(a)

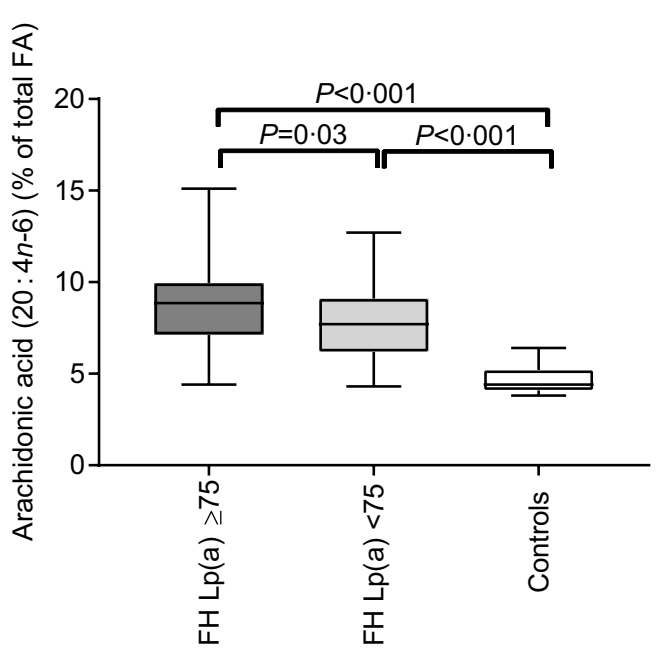

(c)
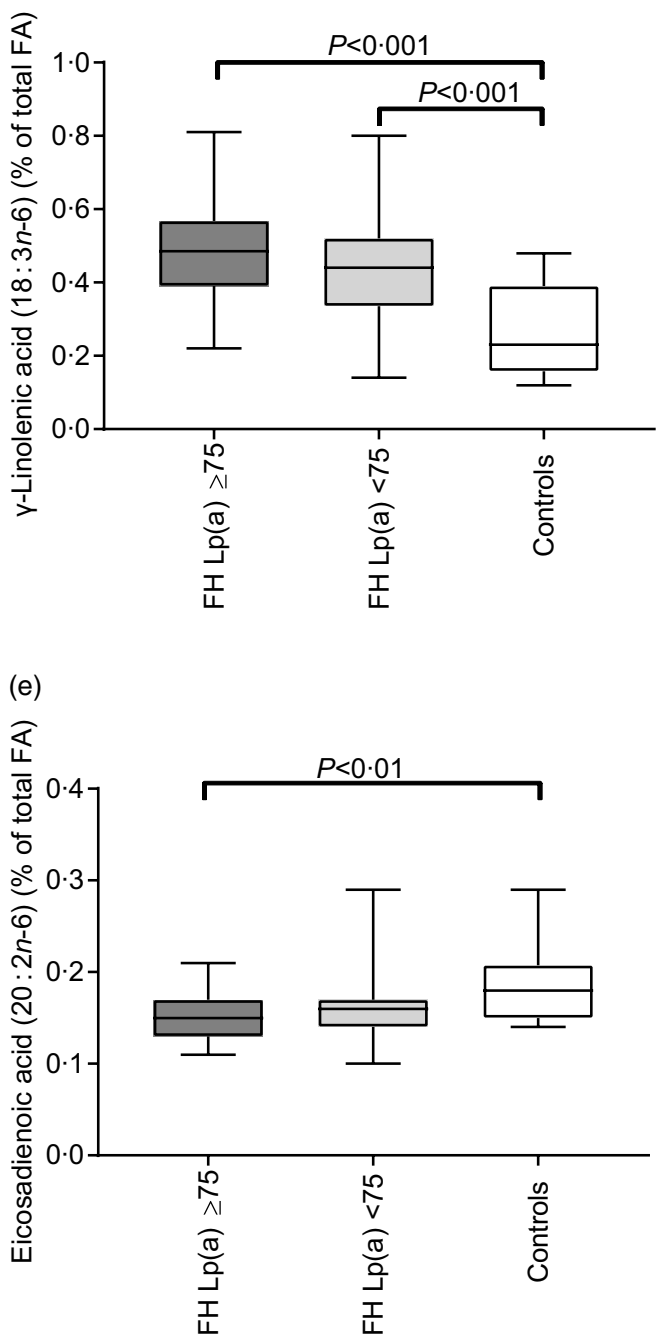

10 (e)

(b)

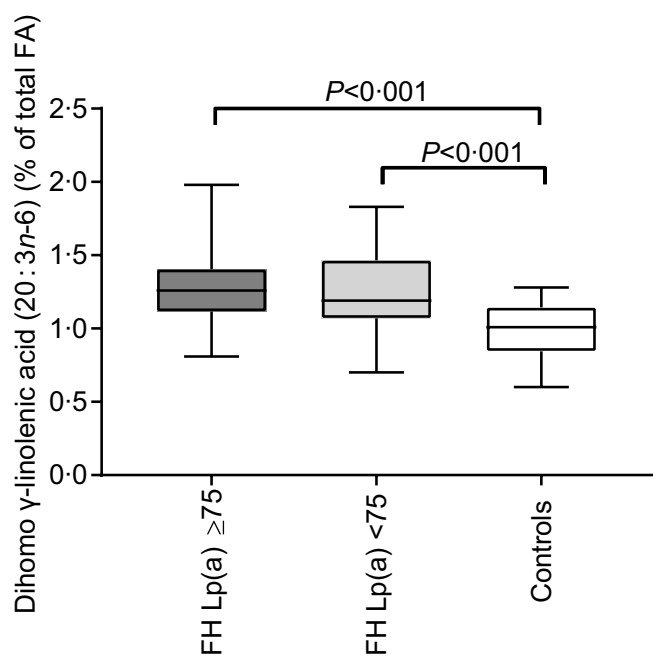

(d)

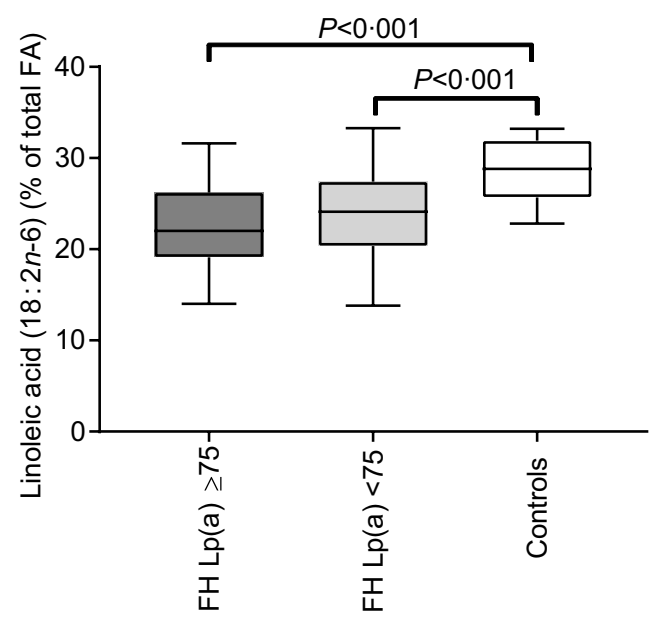


(a)

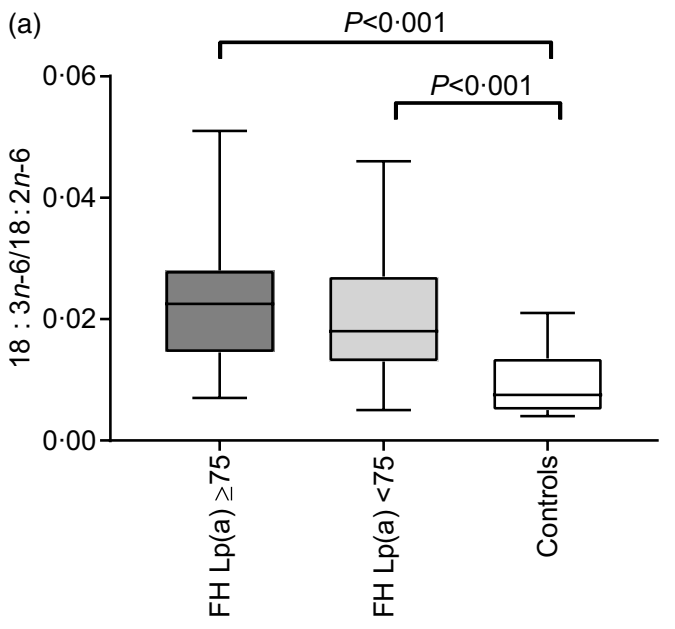

(c)

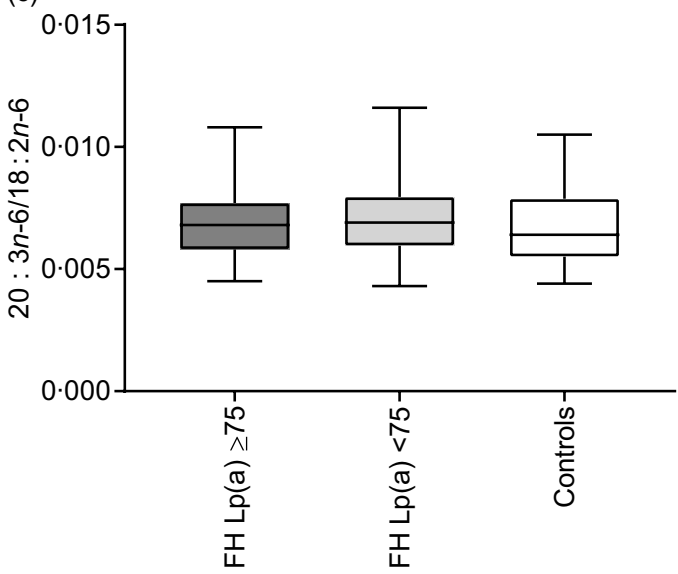

(b)

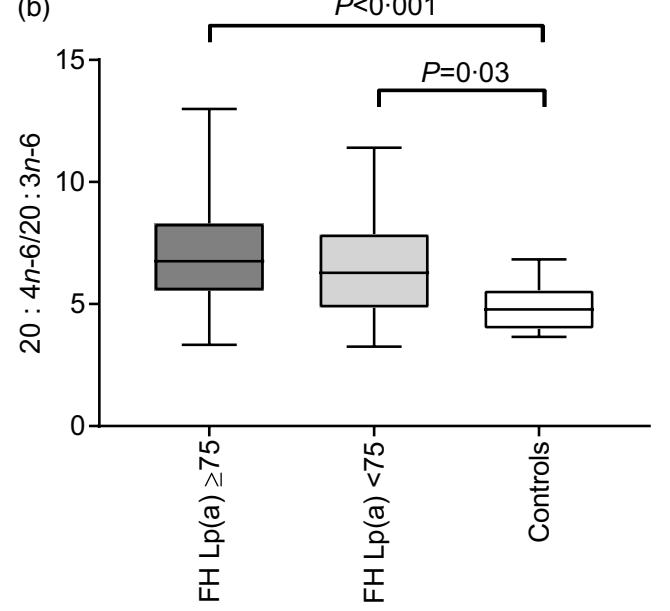

Fig. 2. Estimated ratios as surrogate markers of $\Delta-6$ desaturase (a), $\Delta-5$ desaturase (b) and elongase 5 (c) in familial hypercholesterolaemia (FH) subjects with ( $n 68)$ or without $(n 77)$ elevated lipoprotein(a) (Lp(a)) and healthy controls $(n 14)$. The ratios were calculated as product divided by precursor as indicated on the $y$-axis. Data were analysed by the Kruskal-Wallis test with Bonferroni-corrected post hoc comparisons between the groups when significant and given as median (minimum-maximum).

the FH subjects without elevated $\mathrm{Lp}(\mathrm{a})$ than in the healthy controls $(P=0 \cdot 02)$. A few significant differences between the groups were also observed among the plasma levels of MUFA and SFA (Table 2).

In order to investigate in detail the $n$ - 6 PUFA pathway, we estimated the ratios of certain $n-6$ PUFA (as described in methods). The ratios $18: 3 n-6 / 18: 2 n-6$ and $20: 4 n-6 / 20: 3 n-6$ were higher in both $\mathrm{FH}$ groups compared with the healthy controls $(P<0.05$ for all; Fig. 2(a)).

\section{Dietary intake and dietary pattern}

FH subjects with elevated Lp(a) had higher intake of dietary fibre (E \%) and lower intake of cholesterol (mg) compared with $\mathrm{FH}$ subjects without elevated $\mathrm{Lp}(\mathrm{a})$, and lower intake of transFA (E \%) compared with healthy controls $(P=0.03$ for all; Table 3). There was an overall significant difference in SFA intake between the three groups $(P=0 \cdot 03)$; however, no significant differences were observed in the pairwise comparisons with Bonferroni adjustment. We found no significant differences between the three groups regarding MUFA, total PUFA, $n-3$ or n-6 intake (E \% for all; Table 3). In order to explain the differences in dietary intake between the $\mathrm{FH}$ groups, we explored the dietary patterns of $\mathrm{FH}$ subjects with and without elevated Lp(a). Compared with FH subjects without elevated Lp(a), FH subjects with elevated Lp(a) demonstrated lower intake of cakes $7.9(2 \cdot 1-18.8) v .13 .6(7.5-21.4) \mathrm{g} / \mathrm{d} P=0.006$; sweets $12 \cdot 0(4 \cdot 6-29 \cdot 1) v .18 \cdot 8(12 \cdot 0-38 \cdot 2) \mathrm{g} / \mathrm{d} P=0 \cdot 02$, and non-significantly lower intake of cheese $22 \cdot 2(12 \cdot 7-35 \cdot 1)$ v. $28.2(16 \cdot 0-49 \cdot 2) \mathrm{g} / \mathrm{d} P=0 \cdot 08$. There was no difference in the total intake of food $(\mathrm{g} / \mathrm{d})$ between the $\mathrm{FH}$ groups (data not shown).

\section{Multivariable analyses}

The results of multiple linear regression analyses of intake of $n-6$ PUFA in relation to plasma AA among FH subjects ( $n$ 139) were unchanged after adjustment for age, sex and BMI (crude regression coefficients: 0.32 (95\% CI 0.05, 0.60), P=0 02 and adjusted regression coefficients: 0.32 (95\% CI $0.05,0.59), P=0.02$, for every $\mathrm{E} \%$ change in $n-6$ PUFA). Furthermore, the results of multiple linear regression analyses of intake of linoleic acid in relation 
Table 3. Intake of energy and macronutrients*

(Medians and 25th-75th percentiles)

\begin{tabular}{|c|c|c|c|c|c|c|c|c|c|c|c|c|c|}
\hline & \multicolumn{4}{|c|}{ FH subjects } & \multirow{2}{*}{\multicolumn{2}{|c|}{$\begin{array}{l}\text { Healthy controls } \\
\qquad(n 14)\end{array}$}} & \multirow[b]{3}{*}{$P \dagger$} & \multirow{2}{*}{\multicolumn{3}{|c|}{ Unadjusted }} & \multirow{2}{*}{\multicolumn{3}{|c|}{ Adjusted }} \\
\hline & \multicolumn{2}{|c|}{$\begin{array}{c}\mathrm{Lp}(\mathrm{a}) \geq 75 \mathrm{nmol} / \mathrm{l} \\
\quad(n 66)\end{array}$} & \multicolumn{2}{|c|}{$\begin{array}{c}\mathrm{Lp}(\mathrm{a})<75 \mathrm{nmol} / \mathrm{l} \\
\quad(n 73)\end{array}$} & & & & & & & & & \\
\hline & Median & Percentiles & Median & Percentiles & Median & Percentiles & & $P \neq$ & $P \S$ & $P \|$ & $P \neq$ & $P \S$ & $P \|$ \\
\hline Energy (kJ) & 9187 & $7588-10796$ & 9752 & $8466-11468$ & 10400 & $9334-12710$ & 0.08 & & & & & & \\
\hline Protein (E \%) & 17.9 & $16.5-19.5$ & $17 \cdot 8$ & $16 \cdot 2-19 \cdot 3$ & $18 \cdot 7$ & $15 \cdot 3-19 \cdot 7$ & 0.91 & & & & & & \\
\hline Fat (E \%) & $31 \cdot 2$ & $28 \cdot 1-35 \cdot 2$ & $33 \cdot 2$ & $30 \cdot 0-35 \cdot 8$ & $33 \cdot 3$ & $31 \cdot 5-35 \cdot 4$ & 0.21 & & & & & & \\
\hline SFA (E \%) & $9 \cdot 1$ & $7 \cdot 6-10 \cdot 3$ & 9.5 & $8 \cdot 3-11 \cdot 6$ & $10 \cdot 0$ & $9 \cdot 1-11 \cdot 8$ & 0.03 & 0.03 & 0.03 & 0.41 & 0.09 & 0.09 & 1.00 \\
\hline TFA (E \%) & 0.2 & $0.1-0.2$ & 0.2 & $0.1-0.3$ & 0.3 & $0.2-0.3$ & 0.03 & 0.28 & 0.01 & 0.06 & 0.84 & 0.03 & 0.18 \\
\hline cis-MUFA (E \%) & $12 \cdot 1$ & $10 \cdot 6-14 \cdot 1$ & $12 \cdot 9$ & $11 \cdot 6-14 \cdot 0$ & $12 \cdot 8$ & $11.5-13.6$ & 0.43 & & & & & & \\
\hline cis-PUFA (E \%) & $6 \cdot 9$ & $6 \cdot 0-8.0$ & $6 \cdot 8$ & $5 \cdot 8-7 \cdot 8$ & $6 \cdot 7$ & $5 \cdot 6-7.6$ & 0.64 & & & & & & \\
\hline$n-3$ PUFA (E \%) & 1.8 & $1.4-2 \cdot 4$ & 1.9 & $1 \cdot 2-2 \cdot 4$ & 18 & $12-22$ & 0.72 & & & & & & \\
\hline$n-6$ PUFA (E \%) & $4 \cdot 8$ & $4 \cdot 1-5 \cdot 6$ & $4 \cdot 8$ & $4 \cdot 1-5 \cdot 4$ & 47 & $41-60$ & 0.95 & & & & & & \\
\hline Linoleic acid (E \%) & 4.7 & $3.9-5.5$ & 4.7 & $4 \cdot 0-5 \cdot 3$ & 4.6 & $4.0-5.9$ & 0.93 & & & & & & \\
\hline Arachidonic acid (E \%) & 0.05 & $0.04-0.07$ & 0.06 & $0.05-0.07$ & 0.06 & $0.04-0.07$ & 0.16 & & & & & & \\
\hline Carbohydrates (E \%) & 44.0 & $39 \cdot 5-49 \cdot 3$ & 43.4 & $39 \cdot 4-46 \cdot 8$ & $43 \cdot 3$ & $41.5-46.8$ & 0.64 & & & & & & \\
\hline Starch (E \%) & $21 \cdot 8$ & $18 \cdot 5-26 \cdot 4$ & $21 \cdot 6$ & $17 \cdot 9-24 \cdot 6$ & $23 \cdot 8$ & $20 \cdot 3-30 \cdot 7$ & 0.19 & & & & & & \\
\hline Dietary fibre (E \%) & $2 \cdot 9$ & $2.5-3.5$ & $2 \cdot 6$ & $2 \cdot 2-3 \cdot 0$ & 27 & $25-28$ & 0.03 & 0.01 & 0.19 & 0.6 & 0.03 & 0.57 & 1.00 \\
\hline Monodisaccharides (E \%) & $18 \cdot 0$ & $14 \cdot 7-22 \cdot 0$ & $18 \cdot 1$ & $15 \cdot 3-22 \cdot 5$ & $16 \cdot 7$ & $15 \cdot 0-19 \cdot 7$ & 0.52 & & & & & & \\
\hline Sugar (E \%) & $3 \cdot 8$ & $2 \cdot 4-6 \cdot 4$ & $4 \cdot 7$ & $3 \cdot 3-6 \cdot 8$ & 4.5 & $2 \cdot 3-5 \cdot 5$ & 0.08 & & & & & & \\
\hline Alcohol (E \%) & $2 \cdot 6$ & $1 \cdot 1-4 \cdot 1$ & $2 \cdot 4$ & $1 \cdot 0-4 \cdot 6$ & $1 \cdot 3$ & $0.9-5 \cdot 1$ & 0.82 & & & & & & \\
\hline Cholesterol (mg) & 232 & $180-321$ & 289 & $230-370$ & 268 & 194-331 & 0.03 & 0.01 & 0.4 & 0.37 & 0.03 & 1.00 & 1.00 \\
\hline
\end{tabular}

$\mathrm{FH}$, familial hypercholesterolaemia; Lp(a), lipoprotein(a); E \%, energy percentage; TFA, trans-unsaturated fatty acids.

${ }^{*} P$ values from the Mann-Whitney $U$ test are shown as unadjusted and Bonferroni adjusted.

$\dagger$ Kruskal-Wallis test between the three groups.

¥ Mann-Whitney $U$ test between FH subjects $L p(a) \geq 75 \mathrm{nmol} / \mathrm{l}$ and $\mathrm{FH}$ subjects $\mathrm{Lp}(\mathrm{a})<75 \mathrm{nmol} / \mathrm{l}$.

§ Mann-Whitney $U$ test between FH subjects $L p(a) \geq 75 \mathrm{nmol} / \mathrm{l}$ and controls.

|| Mann-Whitney $U$ test between FH subjects $\mathrm{Lp}(\mathrm{a})<75 \mathrm{nmol} / \mathrm{l}$ and controls.

to plasma AA among FH subjects ( $n$ 139) were unchanged after adjustment for age, sex and BMI (crude regression coefficients: $0.32(95 \% \mathrm{CI} 0.05,0.59), P=0.02$ and adjusted regression coefficients: $0.32(95 \% \mathrm{CI} 0.05,0.60), P=0.02$, for every $\mathrm{E} \%$ change in linoleic acid). The results of multiple linear regression analyses of intake of AA in relation to plasma AA among $\mathrm{FH}$ subjects ( $n$ 139) were unchanged after adjusting for age, sex and BMI (crude regression coefficients: 4.9 (95\% CI $-11 \cdot 3,21 \cdot 1$ ), $P=0.55$ and adjusted regression coefficients: $0.79(95 \%$ CI $-15 \cdot 8,17 \cdot 3), P=0.93$, for every E \% change in AA).

The results of the logistic regression analyses comparing plasma AA levels between the two $\mathrm{FH}$ groups were unchanged after adjusting for age, sex, BMI, use of PCSK9 inhibitors and cholesterol intake (crude and adjusted ORs were 1.29 (95\% CI 1.08, $1.54), P=0.005$ and 1.31 (95\% CI 1.08, 1.58), $P=0.006$ for every unit change in plasma AA for FH subjects with elevated Lp(a) $v$. FH subjects without elevated Lp(a)).

\section{Discussion}

In the present study, we found that $\mathrm{FH}$ subjects with elevated Lp(a) levels had higher plasma levels of AA compared with FH subjects without elevated Lp(a). Furthermore, both FH subjects with and without elevated Lp(a) had higher plasma levels of AA compared with controls. Additionally, the associations between $n$ - 6 PUFA intake and plasma AA, and between plasma $\mathrm{AA}$ and $\mathrm{Lp}(\mathrm{a})$ in FH subjects, further support our hypothesis of a relationship between $n$-6 PUFA and Lp(a). We propose a novel link between $n-6$ PUFA intake, plasma AA and elevated Lp(a) levels.
AA and other higher $n$-6 PUFA derivatives are derived during desaturation and elongation processes from the precursor and essential FA linoleic acid ${ }^{(28)}$; however, the plasma levels of AA may also depend on other processes such as turnover, oxidation and so on ${ }^{(29)}$. Our results from both univariable and multivariable analyses suggest an association between plasma AA level and plasma Lp(a) level in FH subjects. Furthermore, the multivariable analyses show associations between plasma AA and $n$-6 PUFA intake in FH subjects, whereof dietary linoleic acid, but not AA, as expected, is the $n-6$ PUFA associated with plasma AA level. These results support that linoleic acid, which is the most abundant $n$ - 6 PUFA in the diet, is the driver of the association between dietary n-6 PUFA and plasma level of AA. Collectively, this may support an association between plasma Lp(a) concentration, dietary n-6 PUFA and plasma AA level. Consistent with our results, Hikita et al. have shown higher plasma Lp(a) concentration and lower ratio of EPA:AA in patients with compared with without increased risk of CVD ${ }^{(30)}$. Recently, lipid apheresis was found to reduce linoleic acid and AA in the plasma of hyperlipidaemic patients, concomitant with a reduction in Lp(a) levels, further indicating an indirect link between n-6 PUFA and lipoproteins such as LDL and Lp(a) ${ }^{(31,32)}$. Previously, Li et al. showed that omnivores had both higher Lp(a) levels and higher concentration of AA in serum phospholipids ${ }^{(33)}$, possibly due to the increased direct availability of AA from their meat intake. However, the present study is, to our knowledge, the first to show a link between $n$ - 6 PUFA intake, plasma AA and plasma Lp(a) concentration in FH subjects.

The enzymes $\Delta-6$ desaturase (D6D, rate limiting) and D5D convert linoleic acid to $\gamma$-linolenic acid and dihomo- $\gamma$-linolenic 
acid to AA, respectively, and the ratios $18: 3 n-6 / 18: 2 n-6$ and $20: 4 n-6 / 20: 3 n-6$ may be used as surrogate markers of D6D and $\mathrm{D} 5 \mathrm{D}$ activities, respectively ${ }^{(25)}$. Thus, our results may suggest higher D6D and D5D activity leading to lower plasma levels of linoleic acid and higher levels of downstream $n-6$ PUFA such as $\gamma$-linolenic acid, dihomo- $\gamma$-linolenic acid and AA in FH subjects compared with control subjects. Contrary, plasma levels of eicosadienoic acid were lower in FH subjects compared with controls. The conversion of linoleic acid to eicosadienoic acid is a 'side track' from the direct conversion towards $\mathrm{AA}^{(34)}$, possibly indicating a preferred metabolism towards AA in FH subjects. Desaturases are regulated through the transcription factor, sterol regulatory element binding protein-1c, by dietary fat and cholesterol $^{(35)}$. Lower dietary intake of SFA and cholesterol may potentially be surrogate markers of higher PUFA intake and may thus differentially impact the regulation of D6D in FH subjects with elevated Lp(a). Thus, FA regulation of D6D may also partially explain the previously observed increased plasma levels of $\mathrm{Lp}$ (a) after lifestyle-induced, but not bariatric, weight reduction in the study by Berk et al. ${ }^{(4)}$, further supporting the possible biological link between $\mathrm{Lp}$ (a) and AA. However, the link between $n$-6 PUFA and Lp(a) needs further investigation.

Common for the previously mentioned dietary RCT, the intervention groups lowered the intake of SFA but also increased the intake of carbohydrates ${ }^{(5-8)}$, in particular the dietary fibre intake ${ }^{(6,8)}$. In line with this, we also find a higher intake of dietary fibre between the FH subjects with $v$. without elevated $\mathrm{Lp}(\mathrm{a})$. Hence, it cannot be ruled out that the diet-induced $\mathrm{Lp}(\mathrm{a})$ response may be a synergy effect of lowering SFA/increasing PUFA and increasing dietary fibre. In the present study, it is likely that all FH individuals received the same dietary advices regarding their diagnosis. However, the adherence to a beneficial diet is potentially better in the FH subjects with elevated Lp(a) concentration since they had experienced more events of CVD and were more often treated with PCSK9 inhibitors, potentially increasing the awareness of their increased CVD risk. Data on dietary pattern showed a lower intake of cakes, sweets and cheese in FH subjects with elevated Lp(a) compared with FH subjects without elevated $\mathrm{Lp}(\mathrm{a})$, indicating healthier food choices among FH subjects with elevated Lp(a). Although being mechanistically interesting, the diet-induced increase in plasma Lp(a) concentration is, however, probably less clinically significant since a reduction in approximately $100 \mathrm{mg} / \mathrm{dl}$ (approximately $240 \mathrm{nmol} / \mathrm{l}$ when converted according to Gencer et $\left.a l^{(36)}\right)$ in $\mathrm{Lp}(\mathrm{a})$ in a short-time perspective may be required to reduce the CVD risk with the same magnitude achieved by lowering LDL-cholesterol by $1 \mathrm{mmol} / \mathrm{l}^{(37)}$.

The FA content in phospholipids is dominated by $n$-6 PUFA, in particular linoleic acid and AA, rather than $n-3 \mathrm{PUFA}^{(38)}$. Since Lp(a) transports more than $90 \%$ of oxidised phospholipids (OXPL) in plasma, representing an atherogenic feature ${ }^{(39)}$, and there is an association between a dietary marker of increased PUFA intake and the proportion of different phospholipids ${ }^{(40)}$, it is tempting to speculate that increased levels of AA change the proportion of phospholipids, possibly increasing OXPL. Furthermore, linoleic and AA have been shown to be antagonists of farnesoid X receptor $(\mathrm{FXR})^{(41)}$, and FXR activation has been shown to decrease Lp(a) ${ }^{(42)}$. Thus, n-6 PUFA may inhibit FXR, mediating an increase in $\mathrm{Lp}(\mathrm{a})$ required for transporting increased OXPL, and this small increase in Lp(a) could therefore represent a counteracting and even atheroprotective mechanism mediated by $n-6$ PUFA.

Major strengths of the study are that we show associations between plasma AA and $\mathrm{Lp}$ (a) concentrations in both univariable and multivariable analyses. Further, we included mainly genetically verified $\mathrm{FH}$ subjects with and without elevated Lp(a) with dietary data. A major limitation is the low number of control subjects. The main aim of the study was to compare FH subjects with and without elevated Lp(a) levels; however, we chose to include a small number of healthy controls to have a reference 'point' since a number of the analyses we measured (including the plasma FA profile) did not have established prespecified cut-off points. Other limitations are that the study is explorative and hypothesis generating; the use of FA ratios as markers of enzyme activities since the plasma level of the product and the precursor FA may be biased by synthesis, turnover, oxidation, medication and so on; and measuring plasma FA as relative rather than absolute concentrations. However, a major scope of the study was to explore the relation between Lp(a) concentration and plasma levels of specific FA. Thus, we believe that relative values of FA are more meaningful in the present manuscript. Furthermore, FA in erythrocytes would have been a better marker for long-term diet intake reflecting FFQ data, compared with plasma FA from non-fasting blood samples that mainly reflect short-term dietary intake ${ }^{(43)}$. Nevertheless, the FH patients had generally been followed up at the lipid clinic for years and received dietary counselling as part of their treatment. Furthermore, we have previously shown a more beneficial diet in children and young adults with $\mathrm{FH}$ compared with the general population $^{(44)}$. Also, we recently showed that $87 \%$ of all $\mathrm{FH}$ adults, that had been treated at three Norwegian lipid clinics, received dietary counselling ${ }^{(45)}$. Dietary pattern was measured using a score divided into three categories. LDL-cholesterol levels were lower among those with a diet score in the healthiest category at last visit than in subjects with a score in the most unhealthy category $(3 \cdot 2(1 \cdot 2)$ v. $4 \cdot 4(2 \cdot 1) \mathrm{mmol} / \mathrm{l}, P<0 \cdot 001)$. After follow-up at the lipid clinics, the number of subjects with a diet score in the healthiest diet category doubled. These data are also supported by data from the SAFEHEART-study which showed that adults with FH have healthier dietary habits with lower consumption of SFA compared with non-FH ${ }^{(46)}$. Taken together, this may support the notion that $\mathrm{FH}$ subjects have long-lasting improved dietary habits, which may be reflected in plasma FA even if plasma FA mainly reflect short-term dietary intake.

In conclusion, FH subjects with elevated plasma Lp(a) levels had higher plasma levels of the $n-6$ PUFA AA. Furthermore, dietary $n$-6 PUFA intake was associated with plasma AA, where dietary linoleic acid seemed to be the main driver of the dietary $n$-6 PUFA in this association. Our data suggest a novel link between plasma Lp(a) concentration, dietary $n$ - 6 FA and plasma AA concentration, which may contribute to explain the small diet-induced increase in $\mathrm{Lp}$ (a) levels associated with lifestyle changes. Although the increase may not be clinically relevant, this association may be mechanistically interesting in understanding more of the role and the regulation of $\operatorname{Lp}(\mathrm{a})$. 


\section{Acknowledgements}

We gratefully thank all the subjects participating in this study. We also thank Navida Akther Sheikh and Anne Marte WettingJohansen at the Department of Nutrition, University of Oslo, Norway, and The Medical Laboratory at Oslo University Hospital, Rikshospitalet, for excellent technical assistance.

This study was supported by the University of Oslo, Oslo, Norway, the Norwegian National Advisory Unit on FH, Oslo University Hospital, Oslo, Norway, the Throne-Holst Foundation for Nutrition Research, Oslo, Norway and the South-Eastern Regional Health Authority, Oslo, Norway.

I. N., M. P. B. and K. B. H. conceived and designed research; I. N., M. P. B., L. K. L. Ø., T. U. and K. B. H. conducted research; I. N., L. K. L. Ø., M. B. V. and K. B. H. performed statistical analyses; I. N., M. P. B., L. K. L. Ø., S. M. U., K. R., M. M., J. R. v. L., B. H., P. A., M. B. V. and K. B. H., interpreted results; I. N., M. P. B., M. B. V. and K. B. H were responsible for drafting the manuscript; I. N., M. P. B., M. B. V. and K. B. H. were responsible for final content; all authors read, critically revised and approved the final manuscript.

M. P. B. received research grants and/or personal fees from Amgen, Sanofi, MSD, Boehringer Ingelheim, Mills DA and Kaneka, none of which are related to the content of this manuscript. K. R. received research grants and/or personal fees from Amgen, Mills DA, The Directorate for Health in Norway, The Norwegian Medical Association, Sanofi, Chiesi, Takeda, Bayer, MSD, none of which are related to the content of this manuscript. S. M. U. received research grants and/or personal fees from Mills DA, Tine BA and Rimfrost, none of which are related to the content of this manuscript. J. R. v. L. reports honoraria from Akcea and grants from Aegerion/Amryt, none of which are related to the content of this manuscript. K. B. H. reports grants and/or personal fees from Tine SA, Mills DA, Olympic Seafood, Amgen, Sanofi, Kaneka and Pronova, none of which are related to the content of this manuscript. The other authors have no financial relationships relevant to disclose.

\section{Supplementary material}

For supplementary material/s referred to in this article, please visit https://doi.org/10.1017/S0007114519001600

\section{References}

1. Ellis KL, Boffa MB, Sahebkar A, et al. (2017) The renaissance of lipoprotein(a): brave new world for preventive cardiology? Prog Lipid Res 68, 57-82.

2. Nordestgaard BG, Chapman MJ, Ray K, et al. (2010) Lipoprotein(a) as a cardiovascular risk factor: current status. Eur Heart J 31, 2844-2853.

3. Kronenberg F \& Utermann G (2013) Lipoprotein(a): resurrected by genetics. J Intern Med 273, 6-30.

4. Berk KA, Yahya R, Verhoeven AJM, et al. (2017) Effect of diet-induced weight loss on lipoprotein(a) levels in obese individuals with and without type 2 diabetes. Diabetologia $\mathbf{6 0}$, 989-997.

5. Faghihnia N, Tsimikas S, Miller ER, et al. (2010) Changes in lipoprotein(a), oxidized phospholipids, and LDL subclasses with a low-fat high-carbohydrate diet. J Lipid Res $\mathbf{5 1}$, 3324-3330.

6. Berglund L, Lefevre M, Ginsberg HN, et al. (2007) Comparison of monounsaturated fat with carbohydrates as a replacement for saturated fat in subjects with a high metabolic risk profile: studies in the fasting and postprandial states. Am J Clin Nutr 86, 1611-1620.

7. Ginsberg HN, Kris-Etherton P, Dennis B, et al. (1998) Effects of reducing dietary saturated fatty acids on plasma lipids and lipoproteins in healthy subjects: the DELTA Study, protocol 1. Arterioscler Thromb Vasc Biol 18, 441-449.

8. Silaste ML, Rantala M, Alfthan G, et al. (2004) Changes in dietary fat intake alter plasma levels of oxidized low-density lipoprotein and lipoprotein(a). Arterioscler Thromb Vasc Biol 24, 498-503.

9. Wang DD \& Hu FB (2017) Dietary fat and risk of cardiovascular disease: recent controversies and advances. Annu Rev Nutr 37, 423-446.

10. Mozaffarian D, Micha R \& Wallace S (2010) Effects on coronary heart disease of increasing polyunsaturated fat in place of saturated fat: a systematic review and meta-analysis of randomized controlled trials. PLoS Med 7, e1000252.

11. Hooper L, Martin N, Abdelhamid A, et al. (2015) Reduction in saturated fat intake for cardiovascular disease. Cochrane Database Syst Rev, issue 6, Cd011737.

12. Chowdhury R, Warnakula S, Kunutsor S, et al. (2014) Association of dietary, circulating, and supplement fatty acids with coronary risk: a systematic review and meta-analysis. Ann Intern Med 160, 398-406.

13. Ramsden CE, Zamora D, Majchrzak-Hong S, et al. (2016) Re-evaluation of the traditional diet-heart hypothesis: analysis of recovered data from Minnesota Coronary Experiment (1968-73). BMJ 353, i1246.

14. Sacks FM, Lichtenstein AH, Wu JHY, et al. (2017) Dietary fats and cardiovascular disease: a presidential advisory from the American Heart Association. Circulation 136, e1-e23.

15. Piepoli MF, Hoes AW, Agewall S, et al. (2016) 2016 European guidelines on cardiovascular disease prevention in clinical practice: the sixth joint task force of the European Society of Cardiology and other societies on cardiovascular disease prevention in clinical practice (constituted by representatives of 10 societies and by invited experts) developed with the special contribution of the European Association for Cardiovascular Prevention \& Rehabilitation (EACPR). Eur Heart J 37, 2315-2381.

16. Harris WS, Poston WC \& Haddock CK (2007) Tissue $n-3$ and $n-6$ fatty acids and risk for coronary heart disease events. Atherosclerosis 193, 1-10.

17. Virtanen JK, Wu JHY, Voutilainen S, et al. (2018) Serum n-6 polyunsaturated fatty acids and risk of death: the Kuopio Ischaemic Heart Disease Risk Factor Study. Am J Clin Nutr 107, 427-435.

18. Marklund M, Wu JHY, Imamura F, et al. (2019) Biomarkers of dietary omega- 6 fatty acids and incident cardiovascular disease and mortality: an individual-level pooled analysis of 30 cohort studies. Circulation 139, 2422-2436

19. Ramsden CE, Zamora D, Leelarthaepin B, et al. (2013) Use of dietary linoleic acid for secondary prevention of coronary heart disease and death: evaluation of recovered data from the Sydney Diet Heart Study and updated meta-analysis. BMJ 346, e8707.

20. Zhao JV \& Schooling CM (2019) Effect of linoleic acid on ischemic heart disease and its risk factors: a Mendelian randomization study. BMC Med 17, 61 .

21. Nordestgaard BG, Chapman MJ, Humphries SE, et al. (2013) Familial hypercholesterolaemia is underdiagnosed and 
undertreated in the general population: guidance for clinicians to prevent coronary heart disease: consensus statement of the European Atherosclerosis Society. Eur Heart J 34, 3478-3490.

22. Mundal L, Veierod MB, Halvorsen T, et al. (2016) Cardiovascular disease in patients with genotyped familial hypercholesterolemia in Norway during 1994-2009, a registry study. Eur J Prev Cardiol 23, 1962-1969.

23. Alonso R, Andres E, Mata N, et al. (2014) Lipoprotein(a) levels in familial hypercholesterolemia: an important predictor of cardiovascular disease independent of the type of LDL receptor mutation. J Am Coll Cardiol 63, 1982-1989.

24. Ulven SM, Leder L, Elind E, et al. (2016) Exchanging a few commercial, regularly consumed food items with improved fat quality reduces total cholesterol and LDL-cholesterol: a double-blind, randomised controlled trial. $\mathrm{Br} J$ Nutr 116, 1383-1393.

25. Kawashima A, Sugawara S, Okita M, et al. (2009) Plasma fatty acid composition, estimated desaturase activities, and intakes of energy and nutrient in Japanese men with abdominal obesity or metabolic syndrome. J Nutr Sci Vitaminol (Tokyo) 55, 400-406.

26. Carlsen MH, Lillegaard IT, Karlsen A, et al. (2010) Evaluation of energy and dietary intake estimates from a food frequency questionnaire using independent energy expenditure measurement and weighed food records. Nutr J 9, 37.

27. Graham I, Atar D, Borch-Johnsen K, et al. (2007) European guidelines on cardiovascular disease prevention in clinical practice: full text. Fourth Joint Task Force of the European Society of Cardiology and other societies on cardiovascular disease prevention in clinical practice (constituted by representatives of nine societies and by invited experts). Eur J Cardiovasc Prev Rehabil 14, S1-113.

28. Russo GL (2009) Dietary $n-6$ and $n-3$ polyunsaturated fatty acids: from biochemistry to clinical implications in cardiovascular prevention. Biochem Pharmacol 77, 937-946.

29. Hanna VS \& Hafez EAA (2018) Synopsis of arachidonic acid metabolism: a review. $J$ Adv Res 11, 23-32.

30. Hikita H, Shigeta T, Kimura S, et al. (2015) Coronary artery disease severity and cardiovascular biomarkers in patients with peripheral artery disease. Int J Angiol 24, 278-282.

31. Schmocker C, Kassner U, Kiesler S, et al. (2016) A lipidomic analysis approach in patients undergoing lipoprotein apheresis. Atherosclerosis 249, 30-35.

32. Schmocker C, Kassner U, Ostermann AI, et al. (2017) Effect of different lipid apheresis methods on plasma polyunsaturated fatty acids. Atheroscler Suppl 30, 193-199.
33. Li D, Ball M, Bartlett M, et al. (1999) Lipoprotein(a), essential fatty acid status and lipoprotein lipids in female Australian vegetarians. Clin Sci $\mathbf{9 7}, 175-181$.

34. Lee JM, Lee H, Kang S, et al. (2016) Fatty acid desaturases, polyunsaturated fatty acid regulation, and biotechnological advances. Nutrients $\mathbf{8}, 23$.

35. Nakamura MT \& Nara TY (2004) Structure, function, and dietary regulation of delta6, delta5, and delta9 desaturases. Annu Rev Nutr 24, 345-376.

36. Gencer B, Kronenberg F, Stroes ES, et al. (2017) Lipoprotein(a): the revenant. Eur Heart J 38, 1553-1560.

37. Burgess S, Ference BA, Staley JR, et al. (2018) Association of LPA variants with risk of coronary disease and the implications for lipoprotein(a)-lowering therapies: a Mendelian randomization analysis. JAMA Cardiol 3, 619-627.

38. Spector AA (2001) Plasma free fatty acid and lipoproteins as sources of polyunsaturated fatty acid for the brain. $J \mathrm{Mol}$ Neurosci 16, 159-165; discussion 215-121.

39. Tsimikas S, Tsironis LD \& Tselepis AD (2007) New insights into the role of lipoprotein(a)-associated lipoprotein-associated phospholipase A2 in atherosclerosis and cardiovascular disease. Arterioscler Thromb Vasc Biol 27, 2094-2099.

40. Ruuth M, Nguyen SD, Vihervaara T, et al. (2018) Susceptibility of low-density lipoprotein particles to aggregate depends on particle lipidome, is modifiable, and associates with future cardiovascular deaths. Eur Heart J 39, 2562-2573.

41. Zhao A, Yu J, Lew JL, et al. (2004) Polyunsaturated fatty acids are FXR ligands and differentially regulate expression of FXR targets. DNA Cell Biol 23, 519-526.

42. Hoover-Plow J \& Huang M (2013) Lipoprotein(a) metabolism: potential sites for therapeutic targets. Metab Clin Exp 62, 479-491.

43. Hodson L, Skeaff CM \& Fielding BA (2008) Fatty acid composition of adipose tissue and blood in humans and its use as a biomarker of dietary intake. Prog Lipid Res 47, 348-380.

44. Torvik K, Narverud I, Ottestad I, et al. (2016) Dietary counseling is associated with an improved lipid profile in children with familial hypercholesterolemia. Atherosclerosis 252, 21-27.

45. Bogsrud MP, Graesdal A, Johansen D, et al. (2019) LDLcholesterol goal achievement, cardiovascular disease, and attributed risk of $\mathrm{Lp}$ (a) in a large cohort of predominantly genetically verified familial hypercholesterolemia. J Clin Lipidol 13, 279-286.

46. Arroyo-Olivares R, Alonso R, Quintana-Navarro G, et al. (2019) Adults with familial hypercholesterolaemia have healthier dietary and lifestyle habits compared with their non-affected relatives: the SAFEHEART study. Public Health Nutr 22, 1433-1443. 